\title{
Anesthesia Related Complications of Gastrointestinal Endoscopies; A Retrospective Descriptive Study
}

\author{
Ahmad Hormati ${ }^{1,2}$, Abolfazl Mohammadbeigi ${ }^{3}$, Seyed Mojtaba Mousavi ${ }^{4}$, Mohammad Saeidi ${ }^{4}$, \\ Hamed Shafiee ${ }^{4}$, Reza Aminnejad ${ }^{4, *}$
}

1. Gastroenterology and Hepatology Disease Research Center, Qom University of Medical Sciences, Qom, Iran

2. Gastrointestinal and Liver Disease Research Center, Iran University of Medical Sciences, Firoozgar Hospital, Tehran, Iran

3. Department of Epidemiology, Qom University of Medical Sciences, Qom, Iran

4. Department of Anesthesiology and Critical Care, Qom University of Medical Sciences, Qom, Iran

\section{* Corresponding Author:}

Reza Aminnejad, MD Assistant Professor of Anesthesiology, Qom University of Medical Sciences, Qom, Iran

Telefax: + 982536122053

Email: r.aminnejad@yahoo.com

Received: 09 Feb. 2019

Accepted: 16 Jun. 2019

\section{ABSTRACT}

\section{BACKGROUND}

Gastrointestinal endoscopic procedures are widely used for diagnostic and therapeutic measures. Analgesia and sedation/anesthesia are inseparable parts of these studies and their related complications are inevitable.

\section{METHODS}

In a retrograde descriptive study in Shahid Beheshti Hospital, affiliated to Qom University of Medical Sciences, Qom, Iran from December 2014 to December 2018, we gathered information regarding common anesthesia related complications and analyzed them.

\section{RESULTS}

44659 procedures were performed during the study period and records of 21342 men $(47.79 \%)$ and 23317 women $(52.21 \%$ ) were evaluated. Hemodynamic instability $(9998 ; 22.39 \%)$, dysrhythmia (1600; 3.58\%), desaturation (608; $1.36 \%)$, prolonged apnea (34; $0.08 \%)$, aspiration (43; $0.10 \%)$, postoperative nausea and vomiting (PONV) $(636 ; 1.42 \%)$, headache $(106 ; 0.24 \%)$, delirium $(51$; $0.11 \%)$, aphasia $(1 ; 0.00 \%)$, masseter muscle spasm $(1 ; 0.01 \%)$, myocardial infarction $(2 ; 0.00 \%)$, and death $(5 ; 0.01 \%)$ were seen in the patients.

\section{CONCLUSION}

Sedation/anesthesia is enough safe in gastrointestinal endoscopic procedures to enhance the patients' satisfaction and cooperation. If anesthesia with spontaneous breathing and unsecure airway is selected for this purpose, vigilance of anesthesia provider will be the key element of uneventful and safe procedure.

KEYWORDS:

Analgesia, Anesthesia, Endoscopy, Sedation, Patient Safety, Patient Satisfaction

Please cite this paper as:

Hormati A, Mohammadbeigi A, Mousavi SM, Saeidi M, Shafiee H, Aminnejad R. Anesthesia Related Complications of Gastrointestinal Endoscopies; A Retrospective Descriptive Study. Middle East J Dig Dis 2019;11:147-151. doi: 10.15171/mejdd.2019.141.

\section{INTRODUCTION}

Gastrointestinal studies in a large group of patients need variable degrees of analgesia and sedation/anesthesia. Performing these kind of workups without thinking about patient safety and comfort can lead to some catastrophic outcomes specially in psychologic aspects of the patient's life. Sedation/anesthesia carries a spectrum of risks from shivering to death. ${ }^{1,2}$ In this regard, choosing a best individualized method of sedation/anesthesia has a great importance. A systematic review of randomized controlled trials of patients undergoing 
esophago-gastro-duodenoscopy and/or colonoscopy showed that $6 \%-11 \%$ of patients experiences hypoxemia and hypotension is occurred in 5\%-7\% ${ }^{3,4}$

Sedation/anesthesia in a remote location has its rules and necessities. Widespread use of basic and advanced monitoring devices has increased safety features of intervention such as intravenous sedation with the final aim of patient safety and comfort. ${ }^{5,6}$

Although pre-procedural anxiety reduction by sedative/anesthetic medications as a minimum modality has a lot of benefits and per se it can facilitate post-procedural pain control, ${ }^{7}$ type of sedation/anesthesia in this wide spectrum of diagnostic or therapeutic procedures, mainly depends on patient's medical condition and anesthesiologist's preference. Regardless of the anesthetic technique, morbidity and mortality during or after the procedure are unignorable possibilities. These complications are mainly cardiovascular and respiratory. The most frequent anesthesia related complication in these group of patients, is hypotension specially in anesthesia induced by rapid injection of hypnotics such as Propofol. ${ }^{5,8,9}$ Although, most of these complication can be managed by the expert anesthesiologist, some of them can be fatal then careful attention and preparation for each possible scenario is prudent.

\section{MATERIALS AND METHODS}

This was a retrospective case series study in Shahid Beheshti Hospital, Qom university of medical sciences, Qom, Iran from December 2014 to December 2018. Data from anesthesia sheets of all patients admitted for gastrointestinal studies including 44659 patients with upper GI endoscopy, enteroscopy, colonoscopy and ERCP (Endoscopic Retrograde CholangioPancreatography) were recorded. Moreover, demographic data were including sex, age and BMI (Body Mass Index). Data extraction conducted by one trained researcher and there were no missing cases regarding to anesthesia complication.

Patients with ASA-PS (American Society of Anesthesiologists-Physical Status) III or more and those who needed any anesthetic technique other than topical pharyngeal anesthesia plus intravenous anesthesia/sedation were excluded. Anesthesia was performed with premedication by midazolam $(30 \mathrm{mcg} / \mathrm{Kg})$ and fentanyl $(1 \mathrm{mcg} /$ $\mathrm{Kg}$ ) and induction by propofol (1 $\mathrm{mg} / \mathrm{Kg}$ over $2-3 \mathrm{~min}-$ utes) to achieve deep sedation level as a routine practice in our department with respect to recently updated guideline of American Society for Gastrointestinal Endoscopy (ASGE). ${ }^{2}$ All patients were monitored by pulse oximetry, 3 lead ECG and automated NIBP (Non-Invasive Blood Pressure) measurements immediately prior to injection of premedication drugs up to 30 minutes after termination of the procedure (in post anesthesia care unit). Supplemental oxygen was delivered to all patients during the procedure via nasal cannula (3 Lit/min). All procedures were performed in endoscopy unit. All procedures were performed by senior endoscopists. After termination of the procedures, patients were observed for anesthesia or procedure related complications for 6 and 24 hours in upper GI endoscopies and the rest of the procedures respectively.

The informed consent was taken from all studied patients and they were signed that form. Presence of any anesthesia related complication was noted carefully from immediately after induction of anesthesia/ sedation up to 30 minute after termination of the procedure. Hemodynamic instability $30 \%$ or more change in pre-procedural blood pressure or mean arterial pressure less than $60 \mathrm{mmHg}$ or $20 \%$ or more change in pulse rate from baseline recordings), cardiac dysrhythmias (any abnormal rhythm other than patient preoperative rhythm), hypoxia ( $\mathrm{SPO}_{2}$ less than $90 \%$ ), gastric content aspiration to respiratory tract, PONV (Post-Operative Nausea and Vomiting), headache, apnea for more than 30 seconds, abnormal mental status 24 hours after the procedure and mortality were recorded.

Data were analyzed in Social Package for Social Sciences (SPSS) software (USA, Inc. 18). Results were expressed as mean $\pm \mathrm{SD}$ or percentage $(\%)$, when appropriate.

\section{RESULTS}

There were 44659 procedures performed during the study time (depicted in table-1).

In table-2 complications are shown. Some of these adverse events are directly consequences of anesthesia and the others are partly attributable to it. Aphasia and masseter spasm are exactly rare events in this group of patients that motivated us to report them independently.

\section{DISCUSSION}

Sedation for GI endoscopic procedures has been accepted for a long time. Reported anesthesia related 
Table 1: Demographic data

\begin{tabular}{lccccc}
\hline Procedure & Male (\%) & Female (\%) & Mean Age (yr) & Mean BMI (Kg/m²) & Total \\
\hline Endoscopy & $12563(49.12)$ & $13012(50.88)$ & $41.2 \pm 11.1$ & $21.5 \pm 8.3$ & 25575 \\
\hline Colonoscopy & $8359(45.87)$ & $9864(54.13)$ & $49.7 \pm 12.2$ & $23.6 \pm 6.4$ & 18223 \\
\hline Enteroscopy & $23(62.16)$ & $14(37.84)$ & $39.2 \pm 9.1$ & $19.3 \pm 3.1$ & 37 \\
\hline ERCP & $397(48.18)$ & $427(51.82)$ & $56.3 \pm 16.1$ & $26.6 \pm 11.5$ & 824 \\
\hline Sum & $21342(47.79)$ & $23317(52.21)$ & & & 44659 \\
\hline ERCP: Endoscopic retrograde cholangiopancreatography & & & &
\end{tabular}

Table 2: Anesthesia-related complications and their prevalence

\begin{tabular}{lcccccccc}
\hline \multirow{2}{*}{ Complication } & \multicolumn{2}{c}{ Endoscopy } & \multicolumn{2}{c}{ Colonoscopy } & \multicolumn{2}{c}{ Enteroscopy } & \multicolumn{2}{c}{ ERCP } \\
\cline { 2 - 8 } & $\mathbf{M ~ ( \% )}$ & $\mathbf{F ~ ( \% )}$ & $\mathbf{M}(\mathbf{\%})$ & $\mathbf{F ~ ( \% )}$ & $\mathbf{M}(\mathbf{\%})$ & $\mathbf{F ~ ( \% )}$ & M (\%) & F (\%) \\
\hline $\begin{array}{l}\text { Hemodynamic } \\
\text { Instability }\end{array}$ & $2814(22.4)$ & $2407(18.50)$ & $2131(25.49)$ & $2328(23.60)$ & $7(30.43)$ & $6(42.85)$ & $141(35.52)$ & $164(38.40)$ \\
\hline Dysrhythmias & $438(3.49)$ & $410(3.15)$ & $394(4.71)$ & $309(3.13)$ & $1(4.34)$ & $1(7.14)$ & $25(6.29)$ & $22(5.15)$ \\
\hline Desaturation & $138(1.10)$ & $121(0.93)$ & $121(1.45)$ & $131(1.33)$ & $1(4.34)$ & $0(0)$ & $64(16.12)$ & $32(7.49)$ \\
\hline $\begin{array}{l}\text { Prolonged } \\
\text { Apnea }\end{array}$ & $3(0.02)$ & $5(0.04)$ & $6(0.07)$ & $11(0.11)$ & 0 & 0 & $4(1.08)$ & $5(1.17)$ \\
\hline Aspiration & $11(0.09)$ & $13(0.10)$ & $3(0.04)$ & $6(0.06)$ & 0 & $1(7.14)$ & $3(0.76)$ & $6(1.40)$ \\
\hline PONV & $231(1.84)$ & $169(1.30)$ & $112(1.34)$ & $113(1.14)$ & 0 & 0 & $3(0.76)$ & $8(1.87)$ \\
\hline Headache & $23(0.18)$ & $33(0.25)$ & $19(0.23)$ & $21(0.21)$ & $1(4.34)$ & $2(14.29)$ & $3(0.76)$ & $4(0.94)$ \\
\hline Delirium & $12(0.09)$ & $13(0.10)$ & $9(0.11)$ & $11(0.11)$ & $1(4.34)$ & 0 & $4(1.08)$ & $1(0.23)$ \\
\hline Aphasia & $1(0.008)$ & 0 & 0 & 0 & 0 & 0 & 0 & 0 \\
\hline Masseter Spasm & 0 & 0 & 0 & $1(0.01)$ & 0 & 0 & 0 & 0 \\
\hline MI & 0 & 0 & $1(0.01)$ & $1(0.01)$ & 0 & 0 & 0 & 0 \\
\hline Death & $1(0.008)$ & 0 & 0 & $1(0.01)$ & 0 & 0 & $1(0.25)$ & $2(0.47)$ \\
\hline
\end{tabular}

MI: Myocardial infarction, PONV: postoperative nausea and vomiting, ERCP: Endoscopic retrograde cholangiopancreatography

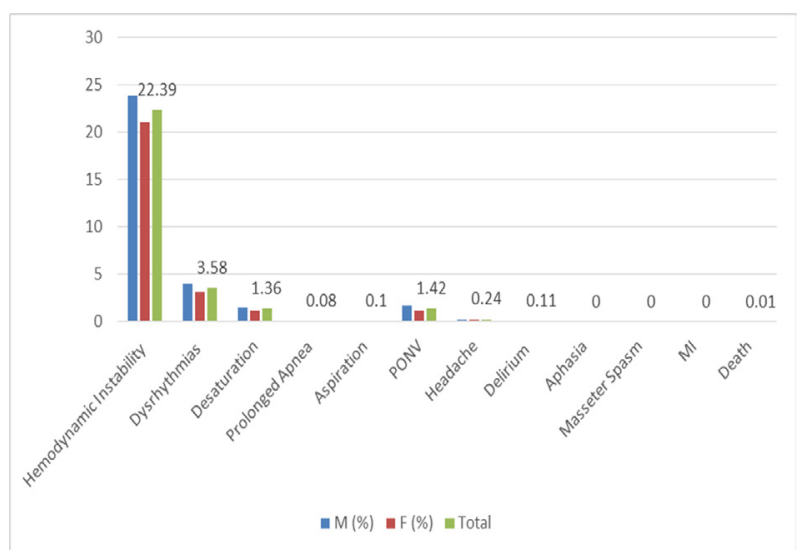

Chart 1: The overall prevalence of anesthesia-related complications in both sexes

complication rates in these procedures mainly depend on sample sizes. Then limited sample sizes in most of studies, can lead to variable results. Trying to have a large group of study patients despite its difficulties, can help us to better understanding the real risks of such a frequently used procedures. In recently published study by Behrens et al. ${ }^{10}$ overall rate of all sedation-associated complications was $0.3 \%$ (depicted in detail in chart- 1 ). This very low rate of adverse events showed that sedation can be implemented safely for a such unpleasant experience for patients with GI problems. Behrens and his colleagues documented that respiratory depression is the most commonly occurred unwanted event in these group of patients. ${ }^{10}$ Whilst in some other studies, hypotension has been introduced as the most common complication. ${ }^{8}$ It's logical that level of anesthesia and drug regimen used for a procedure, maybe the most important factor in determining the relative prevalence of a complication; the deeper the level of anesthesia, the more prevalence of hypotension following injection of common hypnotic drugs specially propofol. 
Nowadays propofol alone is the preferred regimen for sedating patients need GI endoscopic workups. ${ }^{10}$ Hypotension would be the most common adverse event following administration of propofol as a sole anesthetic in GI endoscopic procedures. ${ }^{8}$ In our study this concept was manifested again. Bradycardia as a common dysrhythmia can be another prevalent cardiovascular event in these situations. ${ }^{8}$

When we are talking about anesthesia or sedation in a remote location such as endoscopy room, the greatest concern for anesthesiologists and endoscopists, maybe is the significant fall in oxygen saturation of arterial blood $\left(\mathrm{SPO}_{2}\right)$. Supplementation of inspired air with oxygen can't guarantee the sufficient amount of oxygen in circulation and pulmonary ventilation is a key factor in here. Most of the anesthetic drugs such as hypnotics are respiratory depressant and safe anesthesia mandates careful attention to this aspect of the patient's vital signs. In our study, desaturation was the second most prevalent adverse event that can be directly attributable to anesthesia (after hemodynamic instability) with a rate of $1.52 \%$ and $1.22 \%$ in men and women respectively. These findings are the same as other studies performed with the same drug regimen. ${ }^{8}$ Sharing the upper airway with endoscopists and need for maintaining spontaneous ventilation in these patients can an enough reason for trying to predict at risk cases. ${ }^{11}$ Prolonged desaturation is a great danger for life, so in all cases with prolonged apnea $(0.06 \%$ in men and $0.09 \%$ in women) cessation of the procedure and appropriate interventions were considered in our study.

All of our cases were in appropriate fasting state (at least 8 hours for solid foods), but this preparation can't guarantee complete emptiness of stomach at the time of GI endoscopy. Induction of aesthesia with hypnotics such as propofol, abolishes airway protective reflexes and predisposes patients to the risk of aspiration of regurgitatant gastric contents and increased age and high BMI can increase this risk dramatically. ${ }^{12}$ Rate of aspiration in our study was acceptable and none of our cases suffered from consequences of aspiration in a short term follow-up.

PONV is among the commonest postoperative patient complaints. GI endoscopy per se can predispose patient to postoperative discomfort in gastrointestinal system. Insufflation of GI tract during endoscopic procedures beside pharmacologic effect of anesthetic drugs (specially opioids) can increase the chance of PONV. This phenomenon was the second most prevalent complication after hemodynamic instability in our study. Recent approaches for quality improvement mandate health care providers to think about the common complains and preparing for preventing and resolving them by implementation of multifaceted strategies specially in higher risk patients. ${ }^{13}$

Headache isn't a frequent finding in PACU (Post Anesthesia Care Unit) patients (with the incidence of as low as $1.2 \%) .{ }^{14}$ In our study, the incidence of PACU headache was lower. Reduction of headache incidence in our patients may be due to the use of propofol as a hypnotic medication in all cases. It has been documented in multiple studies that some kind of headaches can be managed well by the use of propofol..$^{15-17}$

There are many hypothetical mechanisms for explaining the occurrence of postoperative delirium. One of them is the direct neurotoxic effect of general anesthetic medications such as propofol ${ }^{18}$ In our study, rate of this adverse event isn't significant.

Occurrence of rare complications such as aphasia ${ }^{18}$ or masseter spasm ${ }^{9}$ repeatedly notify the significance of close observation and implementation of all standard monitoring in endoscopy room. It is important to be aware of the risk of death in an apparently simple procedure such as GI endoscopy. Mortality in this group of patient can be anesthesia related or it can be purely due to procedural complications such as pancreatitis. ${ }^{19,20}$

\section{CONCLUSION}

Sedation/anesthesia is enough safe in gastrointestinal endoscopic procedures to enhance patient satisfaction and cooperation. Anesthesia/sedation with spontaneous breathing of the patients, may need more vigilance by the anesthesia provider than general anesthesia because of the unsecured airway. Applying standards such as operating room standards will help to enhance patient safety.

\section{ACKNOWLEDGMENT}

We thank all anesthesia and endoscopy team members and our colleagues (anesthesiologists and gastroenterologists) in Shahid Beheshti Hospital who without them this study was not possible. 


\section{ETHICAL APPROVAL}

There is nothing to be declared.

\section{CONFLICT OF INTEREST}

The authors declare no conflict of interest related to this work.

\section{REFERENCES}

1. Ferreira AO, Cravo M. Sedation in gastrointestinal endoscopy: Where are we at in 2014? World J Gastrointest Endosc 2015;7:102-9. doi:10.4253/wjge.v7.i2.102.

2. Solhpour A, Jafari A, Hashemi M, Hosseini B, Razavi S, Mohseni G, et al. A comparison of prophylactic use of meperidine, meperidine plus dexamethasone, and ketamine plus midazolam for preventing of shivering during spinal anesthesia: a randomized, double-blind, placebocontrolled study. J Clin Anesth 2016;34:128-35. doi: 10.1016/j.jclinane.2016.03.036.

3. Levy I, Gralnek IM. Complications of diagnostic colonoscopy, upper endoscopy, and enteroscopy. Best Pract Res Clin Gastroenterol 2016;30:705-18. doi:10.1016/j. bpg.2016.09.005.

4. McQuaid KR, Laine L. A systematic review and metaanalysis of randomized, controlled trials of moderate sedation for routine endoscopic procedures. Gastrointest Endosc 2008;67:910-23. doi:10.1016/j.gie.2007.12.046.

5. Moshiri E, Modir H, Yazdi B, Susanabadi A, Salehjafari N. Comparison of the effects of propofol and dexmedetomidine on controlled hypotension and bleeding during endoscopic sinus surgery. Ann Trop Med Public Health 2017;10:721.

6. Mohaghegh T, Yazdi B, Norouzi A, Fateh S, Modir H, Mohammadbeigi A. Effect of intravenous anesthesia with propofol versus isoflurane inhalation anesthesia in postoperative pain of inguinal herniotomy: a randomized clinical trial. Med Gas Res 2017;7:86-92. doi: 10.4103/2045-9912.208511.

7. Mosaffa F, Mohajerani SA, Aminnejad R, Solhpour A, Dabir S, Mohseni GR. Preemptive oral clonidine provides better sedation than intravenous midazolam in brachial plexus nerve blocks. Anesth Pain Med 2016;6:e28768. doi:10.5812/aapm.28768.

8. Amornyotin S, Kachintorn U, Kongphlay S. Anesthetic management for small bowel enteroscopy in a World Gastroenterology Organization Endoscopy Training Center. World J Gastrointest Endosc 2012;4:93-189. doi: 10.4253/wjge.v4.i5.189.

9. Saeidi M, Alikhani R, Hormati A, Sabouri SM, Aminnejad R. Propofol-Induced Masseter Muscle Spasm in a Woman with a Major Depressive Disorder. Anesth Pain Med 2018;8:e78748. doi:10.5812/aapm.78748.
10. Behrens A, Kreuzmayr A, Manner H, Koop H, Lorenz A, Schaefer C, et al. Acute sedation-associated complications in GI endoscopy (ProSed 2 Study): results from the prospective multicentre electronic registry of sedation-associated complications. Gut 2018 pii:gutjnl-2015-311037. doi:10.1136/gutjnl-2015-311037.

11. Goudra BG, Singh PM, Penugonda LC, Speck RM, Sinha AC. Significantly reduced hypoxemic events in morbidly obese patients undergoing gastrointestinal endoscopy: predictors and practice effect. J Anaesthesiol Clin Pharmacol 2014;30:71-7. doi:10.4103/0970-9185.125707.

12. Gemma M, Pasin L, Oriani A, Agostoni M, Palonta F, Ramella B, et al. Swallowing impairment during propofol target-controlled infusion. Anesth Analg 2016;122:48-54. doi:10.1213/ANE.0000000000000796.

13. Pym A, Ben-Menachem E. The effect of a multifaceted postoperative nausea and vomiting reduction strategy on prophylaxis administration amongst higher-risk adult surgical patients. Anaesth Intensive Care 2018;46:185-9. doi:10.1177/0310057X1804600207.

14. Alkandari Z, Kind SL, Spahn DR, Biro P. Early post-anaesthesia recovery parameters-a prospective observational study. Rom J Anaesth Intensive Care 2015;22:103-10.

15. Long BJ, Koyfman A. Benign headache management in the emergency department. J Emerg Med 2018;54:45868. doi:10.1016/j.jemermed.2017.12.023.

16. Krusz JC, Scott V, Belanger J. Intravenous propofol: unique effectiveness in treating intractable migraine. Headache 2000;40:224-30. doi:10.1046/j.1526-4610.2000.00032.x.

17. Giampetro D, Ruiz-Velasco V, Pruett A, Wicklund M, Knipe R. The Effect of Propofol on Chronic Headaches in Patients Undergoing Endoscopy. Pain Res Manag 2018;2018:6018404. doi:10.1155/2018/6018404.

18. Cascella M, Muzio MR, Bimonte S, Cuomo A, Jakobsson JG. Postoperative delirium and postoperative cognitive dysfunction: updates in pathophysiology, potential translational approaches to clinical practice and further research perspectives. Minerva anestesiol 2017;84:246260. doi:10.23736/S0375-9393.17.12146-2.

19. Sarkeshikian S, Hormati A, Ghadir M, Pezeshki Modares M, Alami F. Relative Prevalence of Duodenal Diverticulum and its Association with Pancreatic Biliary Disease in ERCP Patients. J Zanjan Univ Med Sci 2018;26:46-52.

20. Hormati A, Sarkeshikian SS, Alemi F, Ghadir MR. PostERCP Pancreatitis With Normal Serum Amylase Level: A Case Report. Govaresh 2018;23:93-7. 